\title{
Correlation Between Antibiotic Use and Resistance in a Hospital: Temporary and Ward-Specific Observations
}

\author{
I. Willemsen, D. Bogaers-Hofman, M. Winters, J. Kluytmans
}

\begin{abstract}
Objectives: The objectives of this study were to determine (1) the increase in antimicrobial resistance to frequently used antibiotics in the hospital setting over time and (2) the correlation between the amount of use of an antibiotic in a specific medical specialty and the observed resistance to that antibiotic in that specialty.

Method: The total use of antibiotics and the use of ciprofloxacin (CIP), co-amoxicillin + clavulanic acid (AMCL) and firstand second-generation cephalosporins (CEF), respectively, in individual medical specialties were measured between 2001 and 2006 by means of prevalence surveys (two per year). The antimicrobial susceptibility patterns among $E$. coli isolated from hospitalized patients between 2003 and 2006 were obtained from the Laboratory Information System. Trends over time and correlation between use and resistance were calculated.

Results: 6,639 patients were included in the prevalence surveys, of whom $3.0 \%$ (195) were treated with CIP, 9.7\% (642) with AMCL, and 3.5\% (232) with CEF. 4,790 E. coli isolates were obtained from hospitalized patients. Resistance to all antibiotics significantly increased over time, with the regression line showing that the strongest increase in resistance was for CIP (2.6\% per year). There were large variations in antimicrobial use between various medical specialties. A significant correlation was found between the ward-specific prevalence of use and the percentage of resistance for CIP $(R=0.81, p<0.001)$ and AMCL $(R=0.82, p=0.003)$. Conclusion: At the level of individual medical specialties within one hospital, a higher prevalence of antimicrobial use among patients was associated with a significantly higher observed antimicrobial resistance. The use of CIP was associated with a stronger increase in resistance than the use of beta-lactams.
\end{abstract}

Infection 2009; 37: 432-437

DOI 10.1007/s15010-009-8325-Y

\section{Introduction}

The threat to human health posed by antimicrobial resistance is of growing concern [1, 2]. Many commensal and pathogenic organisms have developed resistance to antimicrobial agents [3]. In general, the development of resistance to microorganisms is associated with the amount of use of antimicrobial agents, as shown repeatedly in studies at national and regional levels [4, 5]. The use of antimicrobial agents in the entire hospital correlates with the observed level of resistance [6]. However, the relationship between the amount of use within individual medical specialties of a hospital and the observed level of resistance in this medical specialty has not been clearly established. Harbarth et al. [7] investigated this relationship but was unable to find a significant relation for most of the antibiotics studied. It is important to have a good understanding of the relationship between the amount of use of antibiotics on individual wards and the development of antimicrobial resistance as such information would support targeted intervention programs, such as ward-specific or antimicrobial agent-specific interventions.

The objectives of the study were to determine the increase in resistance over time for the most frequently used antimicrobial agents in our hospital and to determine if the amount of antimicrobial use correlates with the development of resistance in Escherichia coli in individual medical specialties.

\section{Materials and Methods Hospital Setting}

The Amphia hospital is a 1,370-bed teaching hospital, in which all medical specialties are present. In 2005 , there were 40,525 admissions and 265,665 patient-days.

\footnotetext{
I. Willemsen (corresponding author), D. Bogaers-Hofman, J. Kluytmans Laboratory for Microbiology and Infection Control, Amphia Hospital, PO Box 90158, 4800 RK Breda, The Netherlands;

Phone: (+31/76) 595-3015, Fax: -3820, e-mail: iwillemsen@amphia.nl M. Winters

Dept. of Pharmacy, Amphia hospital, Breda, The Netherlands J. Kluytmans

Dept. of Medical Microbiology and Infection Control, VU University Medical Center, Amsterdam, The Netherlands
}

Received: August 21, 2008 - Revision accepted: December 18, 2008 Published online: June 4, 2009 


\section{Use of Antimicrobial Agents}

To determine the use of antimicrobial agents per medical specialty, ten consecutive 1-day prevalence surveys were performed between 2001 and 2006 - two prevalence surveys per year, in the spring and autumn, respectively. The reliability of the prevalence surveys to quantify the use of antimicrobial agents was validated in an earlier study [8]. In this study, prevalence of use corresponds to the prescribed daily doses (PDD). In the prevalence surveys, the use of antimicrobial therapy was measured in all patients who were present in the hospital at 6 a.m. on the day of the survey; patients in daycare, in psychiatric wards, or on hemodialysis were excluded. The three most frequently used antimicrobial agents that are active against Gram-negative rods were included in the analysis: ciprofloxacin (CIP), co-amoxicillin + clavulanic acid (AMCL), and firstand second-generation cephalosporins (CEF), such as cefazolin, cefamandole, and cefuroxime (CFRX). The prevalence of antimicrobial use is expressed as the number of patients using a specific antimicrobial agent on the day of the survey divided by the total number of patients per medical specialty included in this survey.

\section{Antimicrobial Resistance}

The susceptibility patterns of $E$. coli isolated from hospitalized patients between 2003 and 2006 were obtained from the Laboratory Information System (LIS). E. coli was used as the target microorganism because it is the most common Gram-negative microorganism isolated in nosocomial infections in our hospital. Susceptibility patterns before 2003 were not included in our study because prior to 2003 another laboratory method was used. Antimicrobial susceptibility testing was performed with an automated system (Vitek; Biomérieux, Marcy-l'Etoile, France). Interpretation of the antimicrobial susceptibility test results was based on guidelines from the Clinical and Laboratory Standards Institute (CLSI [formerly known as the NCCLS], Wayne, PA) [9]. Repeat isolates cultured from a patient after recovery of the initial isolate were excluded from analysis, unless there was a major difference in the susceptibility pattern. A major difference was defined as $>2$-point difference in susceptibility pattern within 7 days and $>3$-point difference within the whole period (1 point indicates a difference between intermediate and susceptible, or intermediate and resistant).

The percentage of resistance to CIP, AMCL, and CFRX in E. coli isolates was calculated per medical specialty and over time. Analyses were performed twice; in the first analysis, intermediate susceptibility was considered as indicating susceptible, and in the second analysis, intermediate susceptibility was considered as indicating resistant. The trend of resistance to CIP, AMCL, and CFRX over time in the period July 2003 until July 2006 was calculated, including $E$. coli isolates from patients who were hospitalized on the day the culture was taken. We also calculated the correlation between the pooled use of CIP, AMCL, and CEF over the period 2001-2006 and the subsequent pooled resistance to CIP, AMCL, and CFRX in the period July 2003-July 2006 in individual medical specialties. The analyses were repeated with the pooled use of CIP, AMCL, and CFRX over the period July 2003-July 2006 and the resistance rates from the same period. Because CIP is the only fluoroquinolone used in our hospital, only resistance and use of CIP were determined. E. coli infection was considered as hospital acquired if the isolates were obtained $>48 \mathrm{~h}$ after admission.

\section{Data Analyses and Statistics}

Statistical analyses were performed using the Statistical Package for Social Sciences software (SPSS ver. 12.0; SPSS, Chicago, IL).
Trends over time were examined using linear regression analysis. The Pearson correlation coefficient was calculated, and statistical significance was accepted when the chance for coincidence was $<5 \%$.

\section{Results}

A total of 6,639 patients were included in the prevalence surveys between 2001 and 2006, of whom 23\% (1,528) were on antimicrobial therapy. Of these, $3.0 \%$ (195) were treated with CIP, 9.7\% (642) with AMCL, and 3.5\% (232) with CEF. Regression analyses showed no significant changes in the total use of these antibiotics $(p=0.154)$ and in the use of CIP $(p=0.861)$ over the years. However, the use of AMCL $(p=0.009)$ and CEF $(p=0.001)$ did increase significantly during that period. Analysis of the pooled prevalence of antimicrobial use between 2001 and 2006 revealed large variations between various medical specialties, as is shown in table 2. CIP use was the highest in urology $(8.7 \%)$ but not used at all in gynecology; AMCL use was the highest in pulmonary diseases (23.7\%) and lowest in orthopaedic surgery $(2.5 \%)$; CEF use was the highest in urology $(8.2 \%)$ and lowest in cardiology and neurology $(0.9 \%)$.

There were 4,790 E. coli isolates obtained from hospitalized patients between July 2003 and July 2006, which represents $36 \%$ of all Gram-negative microorganisms isolated from patients admitted to the Amphia hospital during this period. The mean number of $E$. coli isolates determined per quarter year was 399 (range 382-420), and no significant change was observed over time (Table 1). There was a significant increase in resistance over time for all antimicrobial agents (CIP, $\mathrm{p}<0.001$; AMCL, $\mathrm{p}=0.031$; CFRX, $\mathrm{p}=0.012$ ). Table 1 shows the number of resistant isolates per quarter year. The increase in microbial resistance against CIP, AMCL, and CEF over time is shown in figure 1 . The strongest increase was observed for CIP, which showed an average annual increase of $2.6 \%$; in comparison, the mean annual increase in microbial resistance to AMCL and CRFX was $1.5 \%$ and $1.9 \%$, respectively.

Table 2 shows the use of CIP, AMCL, and CEF between 2001 and 2006 and the percentage of resistant E. coli isolates between July 2003 and July 2006 in individual specialties. Antimicrobial resistance to CIP was highest in urology $(19.8 \%)$, while resistance to AMCL and CFRX was highest in lung diseases $(11.2 \%)$ and urology $(8.9 \%)$, respectively.

There was a significant relation between the prevalence of use between 2001 and 2006 and the observed resistance to CIP $(\mathrm{R}=0.81, \mathrm{p}=0.005)$ and $\mathrm{AMCL}$ $(\mathrm{R}=0.82, \mathrm{p}=0.003)$ between July 2003 and July 2006 in individual specialties, as shown in figure 2 . A relative low amount of use was associated with a much higher observed resistance to CIP compared to AMCL. There was no significant relation between CEF use and CFRX resistance $(\mathrm{R}=0.61, \mathrm{p}=0.06)$. The outcome of these associations was similar when only the antimicrobial use 


\begin{tabular}{|c|c|c|c|c|}
\hline Quarter & Total isolates (n) & $\begin{array}{l}\text { CIP } \\
\text { Resistant isolates (n) }\end{array}$ & $\begin{array}{l}\text { AMCL } \\
\text { Resistant isolates (n) }\end{array}$ & $\begin{array}{l}\text { CFRX } \\
\text { Resistant isolates (n) }\end{array}$ \\
\hline 1 & 406 & 16 & 17 & 20 \\
\hline 2 & 374 & 21 & 10 & 9 \\
\hline 3 & 398 & 15 & 26 & 14 \\
\hline 4 & 419 & 15 & 21 & 27 \\
\hline 5 & 371 & 12 & 9 & 14 \\
\hline 6 & 392 & 22 & 27 & 17 \\
\hline 7 & 420 & 28 & 23 & 23 \\
\hline 8 & 391 & 30 & 34 & 23 \\
\hline 9 & 419 & 37 & 38 & 33 \\
\hline 10 & 419 & 37 & 24 & 27 \\
\hline 11 & 382 & 40 & 25 & 30 \\
\hline 12 & 399 & 38 & 30 & 23 \\
\hline
\end{tabular}

\begin{tabular}{|c|c|c|c|c|c|c|}
\hline \multirow[t]{2}{*}{ Medical specialty } & \multicolumn{3}{|c|}{ Prevalence use (\%) } & \multicolumn{3}{|c|}{ Resistance (\%) } \\
\hline & CIP & AMCL & CEF & CIP & AMCL & CFRX \\
\hline Urology & 8.7 & 8.7 & 8.2 & 19.8 & 5.0 & 8.9 \\
\hline Geriatrics & 5.1 & 8.5 & 2.6 & 2.9 & 2.9 & 0.5 \\
\hline Pulmonary diseases & 4.2 & 23.7 & 3.1 & 8.9 & 11.2 & 6.9 \\
\hline Orthopaedic surgery & 3.6 & 2.5 & 8.0 & 2.4 & 4.6 & 4.4 \\
\hline Internal medicine & 5.1 & 13.2 & 3.2 & 8.6 & 6.4 & 4.2 \\
\hline Surgery & 3.0 & 11.0 & 4.2 & 4.8 & 6.0 & 7.8 \\
\hline Cardiology & 0.9 & 8.5 & 0.9 & 6.0 & 2.3 & 3.8 \\
\hline Pediatrics & 0.6 & 4.2 & 1.1 & 1.5 & 2.5 & 3.5 \\
\hline Gynecology & 0.0 & 9.0 & 1.3 & 1.6 & 1.6 & 1.6 \\
\hline Neurology & 1.3 & 5.2 & 0.9 & 3.4 & 3.4 & 1.9 \\
\hline
\end{tabular}
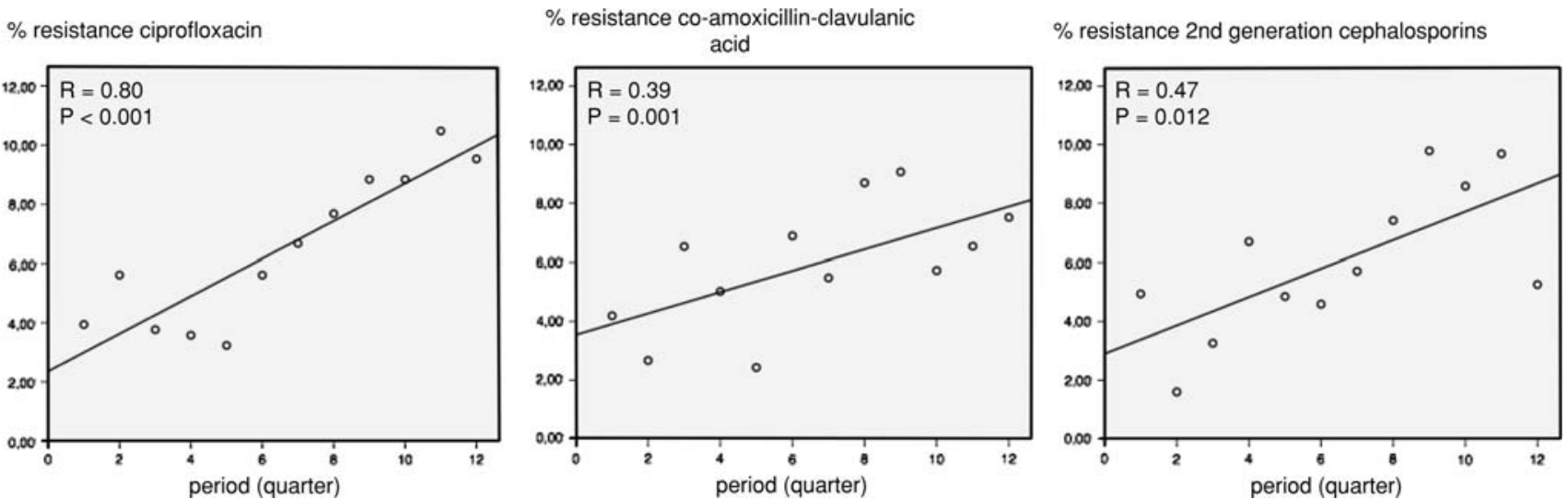

Figure 1. Resistance in E. coli per quarter year to ciprofloxacin, co-amoxicillin + clavulanic acid and cefuroxime over time between 2003 and 2006. 




Figure 2. Correlation between pooled prevalence of use per medical specialty in the period 2001 until 2006 and pooled percentage of resistance of ciprofloxacin, co-amoxicillin + clavulanic acid and cefuroxime in the period July 2003 until July 2006. CIP: ciprofloxacin; AMCL: co-amoxicillin-clavulanic acid.

data from the period July 2003 until July 2006 were included (CIP, $\mathrm{R}=0.75, \mathrm{p}=0.013 ;$ AMCL, $\mathrm{R}=0.80$ / $\mathrm{p}=0.006)$.

The results of an analysis using only the hospitalacquired isolates (isolated at least $48 \mathrm{~h}$ after admission) obtained the same results as the other analyses (CIP, $\mathrm{R}=0.78, \mathrm{p}=0.01 ; \mathrm{AMCL}, \mathrm{R}=0.82, \mathrm{p}=0.004 ; \mathrm{CEF} /$ CFRX, $\mathrm{R}=0.23, \mathrm{p}=0.56$ ).

As a last step, the analyses were repeated including intermediate susceptible isolates to the resistant group. This did not change the overall results (results not shown).

\section{Discussion}

A significant increase in antimicrobial resistance to CIP, AMCL, and CFRX over a relatively short period of time was observed, with the increase of resistance to CIP being stronger than that for AMCL and CFRX. This is a notable result considering that (1) the use of CIP was more or less stable over our study period, whereas the use of AMCL and CEF increased significantly and (2) the density of use of AMCL in the Amphia hospital was more than threefold higher than that of CIP. Based on our results, it would appear that fluoroquinolones are more prone to lead to resistance in microorganisms than beta-lactams. A high level of CIP resistance in relation to its usage has been reported earlier [10-12]. The 2006 European Antimicrobial Resistance Surveillance System report found that only Norway among European countries was able to keep their resistance level against fluoroquinolones in E. coli below $5 \%$, with 11 countries reporting fluoroquinoloneresistant E. coli rates above $25 \%$ [12]. The increase observed in our study is stronger than that observed in other countries in previous studies. In a study carried out in the USA between 1997 and 2000, Lautenbach et al. found an increase in fluoroquinolone resistance in $E$. coli isolated from hospitalized patients of approximately $1.8 \%$ per year [13]. Another study from the USA reported an increase of resistance to CIP among aerobic Gram-negative bacilli in the period 1994-2000 of approximately $1.7 \%$ per year [14]. There is also a trend in the Netherlands towards increasing fluoroquinolone resistance in E. coli (from 3\% in 2000 to 6\% in 2006) [12]. This rapid increase likely represents an evolutionary phase in which resistance is increasing from a low level of resistance towards an equilibrium at a much higher level. We also observed a significant correlation between the amount of use of CIP and AMCL, respectively, and antimicrobial resistance rate to these two antibiotics in individual medical specialties (CIP, $\mathrm{R}=0.81, \mathrm{p}=0.005$; AMCL, $R=0.82, p=0.003$ ). For CFRX, no significant correlation between the amount of use (CEF) and the observed resistance to CFRX was found. A possible explanation for this is that first- and second-generation cephalosporins are frequently used for antibiotic prophylaxis. The dosing regimes for prophylaxis (short duration and relatively high dosage) are different from therapeutic applications.

The main conclusion that we draw from this study is that the use of quinolones is associated with more rapid development of resistance than the use of beta-lactam antibiotics.

Is this conclusion justified considering the methods that were used in this study?

First, to determine the correlation between use and resistance, we made the assumption that antimicrobial usage precedes the development of resistance. From a theoretic point of view, this assumption is logical and is supported by several publications that show a delay between the use of antibiotics and the development of resistance [15]. For resistance in Streptococcus pneumoniae, Van Eldere et al. [16] found a lag period of 1 year between antibiotic use and observed resistance. In our study, a time period of 2 years was used. The analyses were then repeated, excluding the data on usage from 2001 to 2002. The findings from both analyses were similar.

Second, for the analysis of the individual specialties, we pooled the E. coli isolates and the use of antimicrobial agents. It would be interesting to break down the numbers per year, but this did not result in a useful analysis due to the fact that the numbers per subgroup were too small. During the study period no changes were made in the antimicrobial stewardship policies in our hospital and interventions to improve the use of antimicrobials started only after the study was completed.

Third, the influence of antimicrobial use outside the hospital on antimicrobial resistance was not taken into account. Partial adjustment was achieved by includ- 
ing only the hospital-acquired isolates, which resulted in similar conclusions. However, this method will not entirely control for the effect of antimicrobial use in the community $[17,18]$.

Finally, because all data were acquired from one hospital, the emergence of a resistant clone could influence the results of the study. In another study that we recently performed, all resistant $E$. coli strains from hospitalized patients were routinely typed using amplified fragment length polymorphism. A highly heterogeneous pattern was found, and there was not a single clone that had spread in our hospital.

Upon weighing these four different aspects, we consider our conclusion justified. The question that arises next is whether this process of development of resistance is reversible. Oteo et al. [19] investigated the evolution of community use of fluoroquinolones and trimethoprim-sulfamethoxazole (SXT) in comparison with the development of resistance to CIP and SXT in invasive community-acquired $E$. coli infections. Both the use and resistance of fluoroquinolones increased during the study period (2001-2003). However, although SXT use was strongly reduced from 1985 to 2003, SXT resistance showed only a slight decrease between 2001 and 2003. The authors speculate that this is most likely because SXT resistance in $E$. coli is often transferred by resistance genes that share other resistance mechanisms. Therefore, the decreased use of SXT may be compensated for by the use of other agents that also select for SXT resistance [20]. These mechanisms make it very difficult to reverse the process of antimicrobial resistance once it has been established. At the present time, it is unclear whether resistance, once it has been established, can be reversed. Therefore, it is of utmost importance to prevent the development of resistance in an early stage.

The correlation between hospital-wide antimicrobial use and fluoroquinolone resistance among inpatients has been described previously [13, 21, 22]. Our study shows that even at the level of individual medical specialties within one hospital, a higher prevalence of CIP use is associated with the observed rate of resistance. Based on our findings, we feel that it is justified to develop targeted interventions on specific wards. Furthermore, since the use of CIP has been shown to be associated with a stronger increase in antimicrobial resistance over time than the use of beta-lactams, a more restrictive use of fluoroquinolones should be a priority in antimicrobial prescription improvement programs.

\section{Acknowledgments}

This study was funded by the Amphia hospital Breda/Oosterhout.

\section{Conflict of interest statement}

The authors declare that there is no actual or potential conflict of interest in relation to this article.

\section{References}

1. Kunin CM: Resistance to antimicrobial drugs. Ann Intern Med 1993; 118: 557-561.

2. Neu H: The crisis in antibiotic resistance. Science 1992; 257: 1064-1073.

3. Lipsitch $M$, Samore $M H$ : Antimicrobial use and antimicrobial resistance: a population perspective. Emerg Infect Dis 2002; 8: 347-354

4. Austin DJ, Kristinsson KG, Anderson RM: The relationship between the volume of antimicrobial consumption in human communities and the frequency of resistance. Proc Natl Acad Sci USA 1999; 96: 1152-1156.

5. Bronzwaer SL, Cars O, Buchholz U, Mölstad S, Goettsch W, Veldhuijzen IK, Kool JL, Sprenger MJ, Degener JE: European antimicrobial resistance surveillance system. A European study on the relationship between antimicrobial use and antimicrobial resistance. Emerg Infect Dis 2002; 8: 278-282.

6. Binkley S, Fishman NO, LaRosa LA, Marr AM, Nachamkin I, Wordell D, Bilker WB, Lautenbach E: Comparison of unit-specific and hospital-wide antibiograms: potential implications for selection of empirical antimicrobial therapy. Infect Control Hosp Epidemiol 2006; 27: 297-682.

7. Harbarth S, Harris A, Carmeli Y, Samore M: Parallel analysis of individual and aggregated data on antibiotic exposure and resistance in gram-negative bacilli. Clin Infect Dis 2001; 33: 1462-1468.

8. Willemsen I, Groenhuijzen A, Bogaers D, Stuurman A, van Keulen P, Kluytmans J: Appropriateness of antimicrobial therapy measured by repeated prevalence surveys. Antimicrob Agents Chemother 2007; 51: 864-867.

9. Clinical and Laboratory Standards Institution. Performance standards for antimicrobial susceptibility testing. Fifteenth information supplement (M100-S15). CLSI, Wayne, 2005.

10. Livermore DM, James D, Reacher M, Graham C, Nichols T, Stephens $\mathrm{P}$, Johnson AP, George RC: Trends in fuoroquinolone (ciprofloxacin) resistance in Enterobacteriaceae from Bacteremias England, Wales 1990-1999. Emerg Infect Dis 2002; 8: 473-478.

11. Goettsch W, van Pelt W, Nagelkerke N, Hendrix MG, Buiting AG, Petit PL, Sabbe L, van Griethuysen AJ, de Neeling AJ: Increasing resistance to fluoroquinolones in Escherichia coli from urinary tract infections in The Netherlands. J Antimicrob Chemother 2000; 46: 223-228.

12. EARSS Annual Report 2006. European Antimicrobial Resistance Surveillance System.

13. Lautenbach E, Strom BL, Nachamkin I, Bilker WB, Marr AM, Larosa LA, Fishman NO: Longitudinal trends in fluoroquinolone resistance among Enterobacteriaceae isolates from inpatients and outpatients, 1989-2000: differences in the emergence and epidemiology of resistance across organisms. Clin Infect Dis 2004; 38: 655-662.

14. Neuhauser MM, Weinstein RA, Rydman R, Danziger LH, Karam G, Quinn JP: Antibiotic resistance among gram-negative bacilli in US intensive care units: implications for fluoroquinolone use. JAMA 2003; 289: 885-888.

15. Goossens H, Ghysels G, Van Laethem Y, De Wit S, Levy J, De Mol $P$, Clumeck N, Butzler JP, Wenzel RP: Predicting gentamicin resistance from annual usage in hospital. Lancet 1986; 2: 804-805.

16. Van Eldere J, Mera M, Miller LA, Poupard JA, Amrine-Madsen H: Risk factors for development of multiple-class resistance to Streptococcus pneumoniae strains in Belgium over a 10-year period: antimicrobial consumption, population density and geographic location. Antimicrob Agents Chemother 2007; 51: 2491-2497. 
17. Garau J, Xercavins M, Rodríguez-Carballeira M, Gómez-Vera JR, Coll I, Vidal D, Llovet T, Ruiz-Bremón A: Emergence and dissemination of quinolone-resistant Escherichia coli in the community. Antimicrob Agents Chemother 1999; 43: 2736-2741.

18. Endtz HP, Ruijs GJ, van Klingeren B, Jansen WH, van der Reyden T, Mouton RP: Quinolone resistance in campylobacter isolated from man and poultry following the introduction of fluoroquinolones in veterinary medicine. J Antimicrob Chemother 1991; 27: 199-208.

19. Oteo J, Lazaro E, de Abajo FJ, Baquero F, Campos J, Spanish members of EARSS: Antimicrobial-resistant invasive Escherichia coli, Spain. Emerg Infect Dis 2005; 11: 546-553.
20. Enne VI, Livermore DM, Stephens P, Hall LM: Persistence of sulphonamide resistance in Escherichia coli in the UK despite national prescribing restriction. Lancet 2001; 357: 1325-1328.

21. Lautenbach E, Metlay JP, Bilker WB, Edelstein PH, Fishman NO: Association between fluoroquinolone resistance and mortality in Escherichia coli and Klebsiella pneumoniae infections: the role of inadequate empirical antimicrobial therapy. Clin Infect Dis 2005; 41: 923-929.

22. Karlowsky JA, Jones ME, Thornsberry C, Friedland IR, Sahm DF: Trends in antimicrobial susceptibilities among Enterobacteriaceae isolated from hospitalized patients in the United States from 1998 to 2001. Antimicrob Agents Chemother 2003; 47: 1672-1680. 